\title{
BADGE, a synthetic antagonist for PPARY, prevents steroid-related osteonecrosis in a rabbit model
}

\author{
Na Yuan ${ }^{1}$, Jia Li ${ }^{2}$, Meng Li ${ }^{2}$, Wenchen $\mathrm{Ji}^{2}$, Zhaogang Ge${ }^{3}$, Lihong Fan ${ }^{4}$ and Kunzheng Wang ${ }^{4}$
}

\begin{abstract}
Background: It was indicated that inhibition of PPARY probably represents a novel therapy for steroid-related osteonecrosis. In this study, we investigated the preventive effects of PPARY inhibition on steroid-related osteonecrosis in a rabbit model.

Methods: Rabbits were randomly divided into three groups (normal group, model group and BADGE group). Osteonecrosis was induced in rabbits in the model group and the BADGE group. The BADGE group also received bisphenol a diglycidyl ether(BADGE), a PPARy antagonist, for 6 weeks.

Results: Histopathological results indicated that rabbits treated with BADGE exhibited significantly reduced osteonecrotic changes, incidence of osteonecrosis and bone marrow adiposity. Furthermore, BADGE-treated rabbits exhibited reduced intraosseous pressure and increased femoral blood perfusion. Micro-computed tomography and bone histomorphometry indicated that the BADGE group exhibited significantly improved bone quality and mineral appositional rate compared with the model group. Furthermore, the BADGE group showed a significant increase in circulating levels of the bone formation marker osteocalcin and reduced levels of the bone resorption marker TRACP. Overall, BADGE-treated rabbits exhibited reduced marrow adiposity concomitant with improved bone formation.
\end{abstract}

Conclusions: In conclusion, these observations demonstrated that pharmacological inhibition of PPARY might represent an effective therapy for steroid-related osteonecrosis in the near future.

Keywords: Osteonecrosis, Adipogenesis, Ppar $\gamma$, Osteogenesis

\section{Background}

Hypertrophy of bone marrow fat cells, which causes increased intraosseous pressure and decreased blood flow, plays crucial roles in the development of steroid-related osteonecrosis [1-3]. Glucocorticoids promote adipogenic differentiation of marrow stromal cells and inhibit their osteogenic differentiation in vitro [4]. The promoted adipogenesis also acts as a predominantly negative regulator of the bone marrow microenvironment [5] and promotes osteoclast differentiation [6, 7]. These features are potentially related to steroid-related osteonecrosis. Core decompression, which reduces the intra-medullary pressure, was formerly the most commonly used method to treat steroid-

\footnotetext{
* Correspondence: happylee_xjtu@163.com

${ }^{2}$ Department of Orthopedics, The First Affiliated Hospital of Xi'an Jiaotong University, 277 West Yanta Road, Xi'an, Shaanxi 710061, People's Republic of China

Full list of author information is available at the end of the article
}

related osteonecrosis of the femoral head. However, the efficacy of the technique is controversial, and it has little effects on inhibiting bone marrow adipogenesis and fat cell hypertrophy [8].

Increasing evidence demonstrates that peroxisome proliferator-activated receptor $\gamma($ PPAR $\gamma)$, which is a specific transcription factor that plays important roles in adipogenic differentiation, is an important factor in the development of steroid-related osteonecrosis [9-11]. In vivo and in vitro studies indicate that steroids increase the expression of the PPAR $\gamma$ gene, which leads to induced adipogenic differentiation and repressed osteogenic differentiation $[10,12]$. Correspondingly, by suppressing PPAR $\gamma$, several methods are employed to prevent steroid-related osteonecrosis $[9,10]$.

Bisphenol a diglycidyl ether(BADGE), a synthetic antagonist for PPARy, inhibits bone marrow adipogenesis both 
in vitro and in vivo $[13,14]$. In addition, BADGE promotes osteoblastogenesis and bone formation, and reduces marrow adiposity [15]. Based on the abovementioned studies, the utilization of BADGE might offer a promise method for preventing steroid-related osteonecrosis by reducing adipogenesis and intraosseous pressure, improving local blood perfusion of the femoral head, inhibting osteoclastogenesis and promoting osteogenesis.

In the present study, we investigated the preventive effects of BADGE on steroid-related osteonecrosis in a rabbit model and explored the mechanism involved.

\section{Methods}

\section{Animals and treatments}

After approval from the Animal Ethical Committee of Xi'an Jiaotong University, 36 28-week-old male New Zealand white rabbits $(4 \pm 0.5 \mathrm{~kg})$ from the Experimental Animal Center of Xi'an Jiaotong University, China were included in this study. All experimental animals were housed under standard conditions.

Rabbits were randomized into 3 groups: the normal group $(N=12)$, the model group $(\mathrm{N}=12)$ and the BADGE group $(\mathrm{N}=12)$. Osteonecrosis of femurs was induced using methods presented in the previously published protocols [16]. Briefly, one injection of $10 \mu \mathrm{g} / \mathrm{kg}$ body weight of lipopolysaccharide (Sigma, St. Louis, MO, USA) was administered intravenously. Twenty-four hours later, three injections of $20 \mathrm{mg} / \mathrm{kg}$ body weight of methylprednisolone (Pfizer, USA) were administered intramuscularly, at a time interval of $24 \mathrm{~h}$. Osteonecrosis gradually developed in femurs 6 weeks after injection of methylprednisolone. In the model group, the rabbits were treated with vehicle. In the BADGE group, the rabbits received treatment for a total of 6 weeks with BADGE(daily intra-peritoneal injection of $10 \mathrm{mg} / \mathrm{kg}$ in $15 \%$ DMSO). The doses and durations of treatment of BADGE were determined based on a previously published study [15]. Body weight of the rabbits were measured each week.

\section{Histopathology}

The rabbits were sacrificed with an overdose of pentobarbital sodium and bilateral thighbones were dissected for hematoxylin and eosin( $\mathrm{HE})$ staining 6 weeks after the last injection of methylprednisolone. Briefly, bone samples were fixed with $10 \%$ neutral-buffered formalin and decalcified with $10 \%$ ethylene diamine tetraacetic acid for 6 weeks. After the samples were embedded in paraffin, the specimens were cut along the coronal plane into $4-\mu \mathrm{m}$-thick sections and stained with HE. The evaluation criteria of osteonecrosis were based on a published study by Yamamoto et al. [17]. During the observation of slides, the following parameters including incidence of osteonecrosis, rate of empty lacunae, average diameter of fat cells and average fat cell area were assessed by two independent authors who were blind according to previous studies [16, 18-20].

\section{Biochemical analysis}

Blood samples were obtained at week 6 of treatment, and blood serum was prepared from each sample by centrifugation for $5 \mathrm{~min}$ at $3000 \mathrm{rpm}$. Serum was stored frozen at $-20{ }^{\circ} \mathrm{C}$. To assess osteoblastic activity, osteocalcin $(\mathrm{OCN})$ which is a serum marker of bone formation, was measured using an immunoradiometric kit (Beifang BioengineeringInstitute, Beijing, China) according to the manufacturers' instructions. Serum TRACP, marker of bone resorption, was quantified using a TRACP assay kit(Nanjing JianchengBioengineering Institute, Nanjing, China).

\section{Western blot analysis}

The bone samples were crushed under liquid nitrogen conditions using mechanical disruption technique. The protein extracts were obtained by incubation in RIPA buffer at $4{ }^{\circ} \mathrm{C}$ for $30 \mathrm{~min}$. Solutions were then centrifuged at $12000 \mathrm{rpm}$ for $20 \mathrm{~min}$ at $4{ }^{\circ} \mathrm{C}$ to remove insoluble material. Protein concentrations were determined using the BCA assay and the samples were stored at $-80{ }^{\circ} \mathrm{C}$. For Western blot analysis, the samples were dissolved in SDS electrophoresis buffer (Bio-Rad, Hercules, CA, USA) and loaded on SDS-PAGE gels and transferred onto a PVDF membrane filters. The filter was blocked for $1 \mathrm{~h}$ and then incubated overnight at $4{ }^{\circ} \mathrm{C}$ with primary antibodies against osteocalcin, Runx2, adipocyte fatty acid binding protein2(aP2) and $\beta$-actin(Santa-Cruz, USA), followed by incubation with the corresponding secondary antibodies. The protein bands were quantitatively analyzed by use of an image analysis system (NIH Image, Version 1.61).

\section{PCR analysis}

After the bone samples were crushed under liquid nitrogen conditions using a mechanical disruption technique, total RNA was extracted using the TRIzol reagent (Invitrogen, CA, USA) according to the manufacturer's specifications. cDNA was synthesized from $2 \mu \mathrm{g}$ of total RNA using the Revert Aid ${ }^{\mathrm{TM}}$ First Strand cDNA Synthesis Kit (Fermentas, Canada) as described by the manufacturer. The relative levels of targeted gene mRNA transcripts were determined by real-time quantitative RT-PCR using SYBR Green 1 PCR master mix (TaKaRa, Japan). The $\Delta \Delta C t$ method was used to quantify the relative expression of each gene. PCR primers with the following sequences were commercially designed by Shengong Co. Ltd., Shanghai, China: GAPDH, forward: 5'-CCACTTTGTGAAGCTCATT TCCT-3', reverse: 5'-TCGTCCTCCTCTGGTGCTCT3'; osteocalcin, forward: 5'-TCACTCTTGTCGCCC TGCT-3', reverse: 5'-CCTCCCTCTTGGACACGAA-3'; 
Runx2, forward: TGACCGCAGACATAATCCAT, reverse: GCCACTTTCGGAACAGAGAT; CCAAT/ enhancer-binding protein $\beta(C E B P \beta)$, forward: CTAC TACGAGGCGGACTGCT, reverse: GTACGGGCTGA AGTCGATG; aP2, forward: CGATAAACTGGTGGT GGAATGC, reverse: CCCGGGCTTATGCTCTTTCA. Real-time PCR was performed for 40 cycles of $95{ }^{\circ} \mathrm{C}$ for $15 \mathrm{~s}$, an annealing temperature $\left(60{ }^{\circ} \mathrm{C}\right.$ for $\mathrm{GAPDH}, \mathrm{CEBP} \alpha$ and aP2, and $65^{\circ} \mathrm{C}$ for osteocalcin and Runx2) for $30 \mathrm{~s}$, and $72{ }^{\circ} \mathrm{C}$ for $30 \mathrm{~s}$.

\section{Micro-computed tomography $(\mu \mathrm{CT})$ analysis}

$\mu \mathrm{CT}$ (eXploreLocus SP, GE, USA) was performed as described previously [20] to non-invasively evaluate bone structure using MS-8 system, which is a high-resolution system with a resolution of $14 \mu \mathrm{m}$ per voxel, 6 weeks after the last injection of methylprednisolone. Briefly, a cylindricalregion of interest (ROI) in the trabecular region of the femoral head bone was selected. Images were reconstructed into 3-D volumes. The bone mineral density (BMD), bone volume fraction (BVF), trabecular separation (Tb.Sp), trabecular thickness (Tb.Th), and trabecular number (Tb.N) were calculated then.

\section{Dynamic histomorphometry analysis}

Tetracycline and calcein labelling was performed to analyse dynamic histomorphometry. Briefly, rabbits were injected intramuscularly with $50 \mathrm{mg} / \mathrm{kg}$ of tetracycline 10 days before sacrifice and $5 \mathrm{mg} / \mathrm{kg}$ of calcein (Sigma) 3 days before sacrifice. Then, the thighbones were dissected out, fixed in $70 \%$ ethanol, dehydrated, and embedded undecalcified in methylmethacrylate. Then, $10-\mu \mathrm{m}$ sections were cut, and the unstained sections were viewed using a fluorescence microscope. The mineral appositional rate (MAR, $\mu \mathrm{m} /$ day) was measured. MAR was defined as the mean distance between two fluorescent labels divided by the number of days between labels.

\section{Measurements of intraosseous pressure}

Intraosseous pressure in the proximal femur of all rabbits was determined at week 6 . The animals were anesthetized with $30 \mathrm{mg} / \mathrm{kg}$ of pentobarbital sodium. A standard lateral approach to expose the proximal aspect of the right femur was made under sterile conditions as described in previous reports $[1,21]$. To create a drill hole, a drill with an outer diameter of $1.0 \mathrm{~mm}$ was inserted from the outer cortex $2.5 \mathrm{~cm}$ distal to the proximal end of the greater trochanter. The hole was then connected to a pressure transducer via a polyethylene catheter filled with heparinized saline. The pressure transducer was connected to a blood pressure amplifier (FY-2; ChengDu; China) for intraosseous pressure measurements. When the pressure turned steady, the steady recording was obtained as the intraosseous pressure. The systemic arterial pressure was measured simultaneously via an arterial catheter in the rabbit's ear.

\section{Blood perfusion}

To analyse blood perfusion, dynamic contrast-enhanced MRIs for the bilateral proximal femora were performed 6 weeks after the injection of MPS using a 3.0 T superconducting system (ACS-NT Intera, Philips Healthcare, The Netherlands) described previously [16, 22]. As the contrast agent, $0.8 \mathrm{mmol}$ per $\mathrm{kg}$ of body weight of gadopentetatedimeglumine (Gd-DTPA, Magnevist, Schering, Berlin, Germany) was rapidly injected manually via a previously placed 21-gauge intravenous catheter in the right ear vein, followed by a $5-\mathrm{mL}$ saline flush. A set of DCE-MRI was obtained soon after Gd-DTPA injection, and the signal intensity (SI) was measured. SI for each femur was plotted against time to create a time-signal intensity curve, and the perfusion index 'maximum enhancement' was calculated. Maximum enhancement was defined as $\left(\mathrm{SI}_{\max }-\mathrm{SI}_{\text {base }}\right) / \mathrm{SI}_{\text {base }} \times 100 \%$. $\mathrm{SI}_{\text {base }}$ was the baseline signal intensity obtained before Gd-DTPA injection, and $\mathrm{SI}_{\max }$ was the maximum signal intensity after Gd-DTPA injection.

\section{Statistical analysis}

All data were presented as the mean \pm standard deviation (SD). One-way analysis of variance was performed to analyse the statistical data, and the Chi-square test was performed for the count data. The SPSS 17.0 software was used for analysis. In all experiments, a value of $p<0.05$ was considered significant.

\section{Results}

1. Treatment of rabbits with PPAR $\gamma$ inhibitors reduced osteonecrotic changes.

No rabbits died in all groups. The body weight change did not differ between the model group and the BADGE group throughout the experimental period. Obvious osteonecrosis was observed in the model group (Fig. 1) with the diffuse presence of empty lacunae or pyknotic nuclei of osteocytes within the bone trabeculae accompanied by surrounding bone marrow cell necrosis or fat cell necrosis. In the BADGE group, slight osteonecrosis was observed. The incidence of osteonecrosis in the BADGE group was 33\% (4/12) which was lower than $83 \%(10 / 12)$ in the model group $(P<0.05)$. In addition, the rate of empty lacunae in the BADGE group was significantly reduced compared with the model group $(P<0.05)$. 


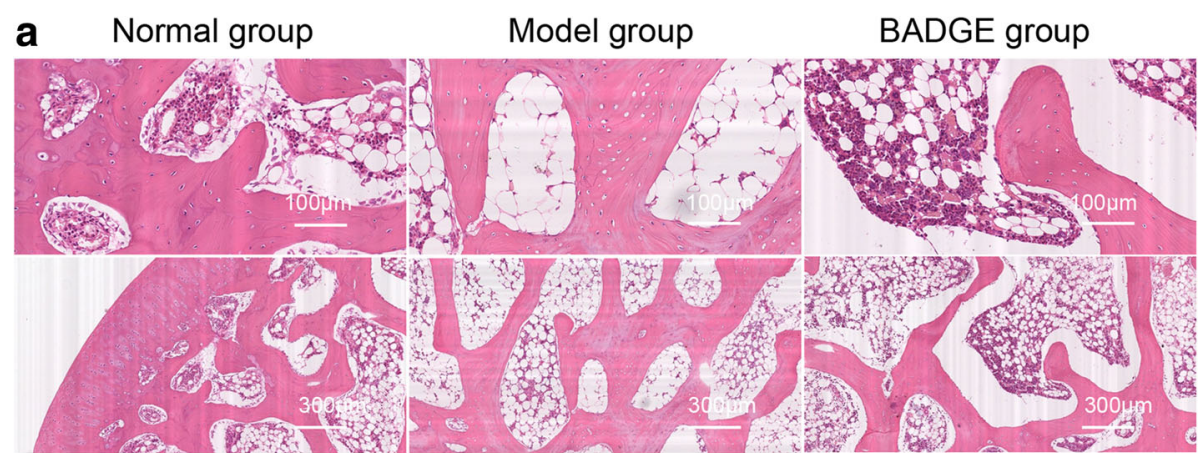

b

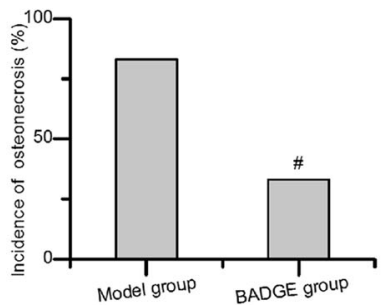

C

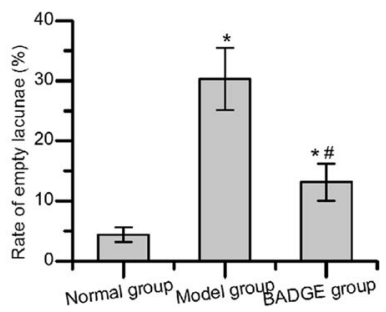

Fig. 1 Treatment with PPARY inhibitors reduced osteonecrotic changes and osteonecrosis incidence. a Histopathological observations. Normal bone tissue and typical osteonecrosis were observed in the normal group and the model group, respectively. In the model group, diffuse osteocytes in the trabeculae exhibited empty lacunae or pyknotic nuclei. In the BADGE group, slight osteonecrosis and fewer empty lacunae were observed. $\mathbf{b}$ A reduced osteonecrosis incidence was observed in the BADGE group compared with the model group $(P<0.05)$. $\mathbf{c}$ The rate of empty lacunae in the BADGE group was significantly reduced compared with the model group, and the significance was statistically significant $(P<0.05, n=5)$. ${ }^{*} P<0.05$ versus normal group; $\# P<0.05$ versus model group

2. BADGE administration decreased bone marrow adiposity and intraosseous pressure, and promoted femoral blood perfusion.

As shown in Fig. 1a, marrow fat was significantly reduced in rabbits treated with BADGE compared with those in the model group. Both the average diameter and average area of fat cells were significantly reduced in the BADGE group compared with the model group (Fig. 2a and b). Furthermore, we detected the local expression of adipogenic factors, including CEBP and $\mathrm{aP} 2$. Both the protein and mRNA level of aP2 and the mRNA level of CEBP in the BADGE group were significantly reduced compared with the model group (Fig. 2c-f). Bone marrow adiposity plays essential roles in increasing intraosseous pressure and reducing blood flow [1-3]. In accordance with the decreased bone marrow adiposity, the intraosseous pressure in the proximal femur of rabbits in the BADGE group declined compared with the model group (Fig. 2g). In addition, blood perfusion to the proximal femora, which was measured by dynamiccontrast enhanced MRIs, was significantly enhanced in the BADGE group compared with the model group (Fig. 2h).

3. BADGE administration increased bone mass and stimulated bone formation

Micro-CT and undecalcified histology (Fig. 3) revealed evidence of increased trabecular bone formation in rabbits treated with the PPARY inhibitor BADGE compared with those in the model group. Micro-CT analysis indicated a significant increase in bone relative to bone mineral density and bone volume fraction in the region of interest (Fig. 3b). Dynamic histomorphometry analysis for tetracycline and calcein labelling revealed increased mineral apposition rates (MAR) in trabecular bone in the rabbits receiving PPARY inhibitors compared with those in the model group (Fig. 3d). The anabolic effect of PPARy inhibitors on bone was further examined by quantifying the circulating concentrations of markers of bone formation (osteocalcin) and bone resorption (TRAP). A significantly increased concentration of osteocalcin was observed in the BADGE group compared with the model group (Fig. 4a). In contrast to increased serum osteocalcin, serum TRAP was reduced in the BADGE group compared with the model group (Fig. 4b). These data suggested coupling of bone formation and resorption. In contrast to the decreased expression of CEBP and aP2, the expression of osteocalcin and Runx2 in the BADGE group was significantly increased compared with the model group (Fig. 4c-f).

\section{Discussion}

In this study, we explored the protective effects of BADGE on steroid-related osteonecrosis in rabbits. Our results indicated that PPARY inhibition prevented steroid-related osteonecrosis, and the effect was in part owing to reduced 


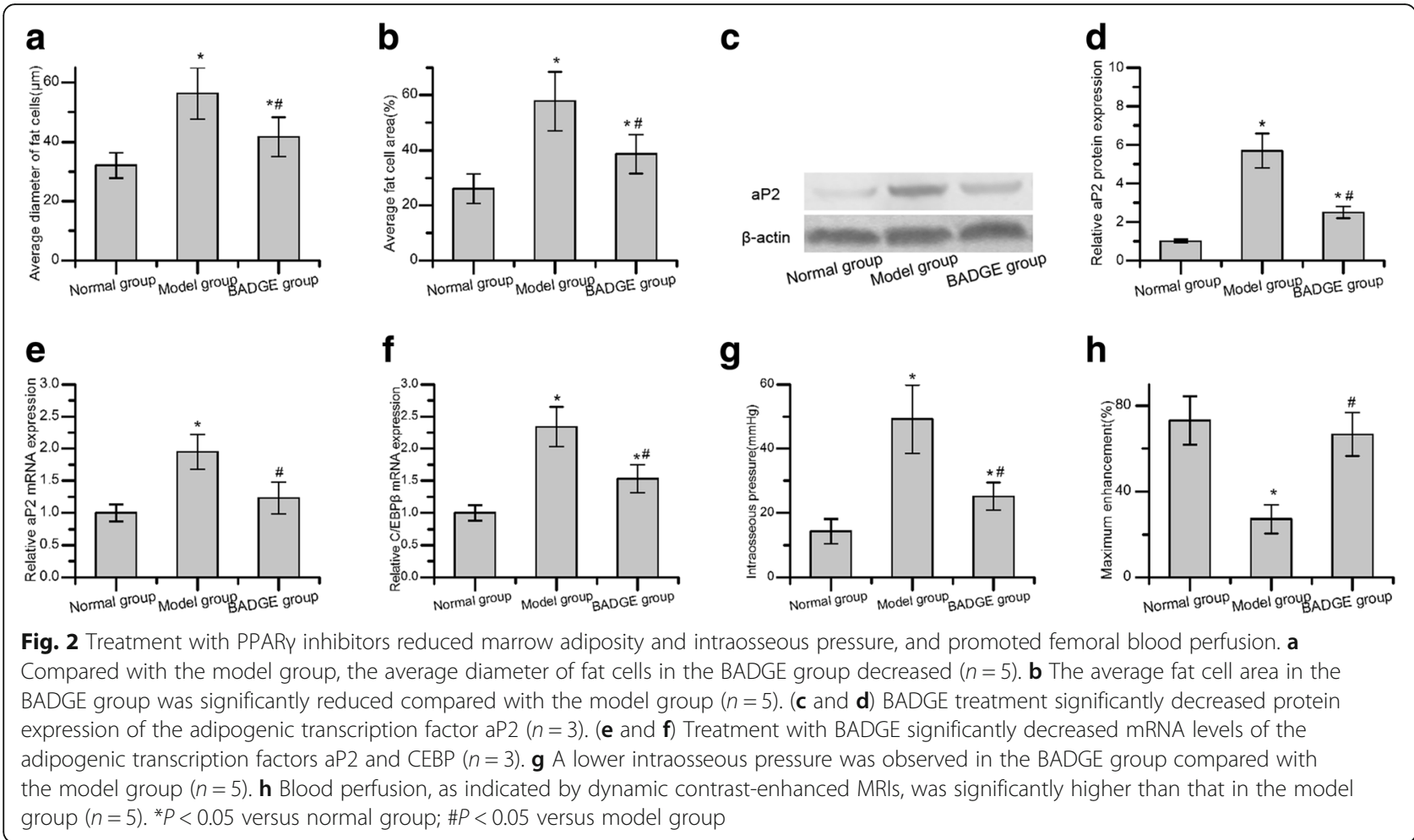

marrow fat infiltration. The reduced bone adiposity was accompanied by an increase in bone mass and femoral blood perfusion and associated with decreased transcription of adipogenic genes and increased transcription of osteogenic genes.

Hypertrophy of fat cells and adipogenic differentiation of BMSCs induced by steroids is potentially associated with steroid-related osteonecrosis [1-3]. Previous studies have demonstrated that PPARY is closely related to the induction of adipogenic differentiation and plays important roles in the development of steroid-related osteonecrosis $[10,12]$. Steroids can also stimulate the expression of PPAR $\gamma$ mRNA both in vivo and in vitro, and increased PPAR $\gamma$ expression can reduce osteogenic differentiation, induce adipogenic differentiation, promote bone marrow adiposity, rise intraosseous pressure, block local blood perfusion, and finally lead to osteonecrosis. Correspondingly, downregulation of PPAR $\gamma$ expression might induce osteogenic differentiation, reduce adipogenic differentiation, inhibit bone marrow adiposity, decrease intraosseous pressure, promote local blood perfusion and eventually prevent osteonecrosis.

As a synthetic antagonist for PPAR $\gamma$, bisphenol a diglycidyl ether (BADGE) inhibits adipogenic differentiation in vitro and prevents bone marrow adiposity in vivo $[13,14]$.

Botolin S and McCabe LR found that BADGE treatment prevents diabetes-induced hyperlipidemia, effectively blocks diabetes type I-induced bone adiposity and reduces the induction of adipogenic gene expression in insulin-deficient diabetic BALB/c mice [14]. Similar results were presented in our study. In the present study, we found that in rabbits treated with both BADGE and steroids, bone marrow adiposity was significantly reduced compared with that in rabbits treated only with steroids. In addition, the expression of adipogenic factors, including CEBP and aP2, was significantly repressed. Furthermore, we detected intraosseous pressure and femoral blood perfusion given that bone marrow adiposity is closely related to intraosseous pressure and blood flow. Similar to the reduced bone marrow adiposity, BADGE administration reduced the intraosseous pressure in the proximal femur and increased blood perfusion to the proximal femora.

A reciprocal relationship exists between osteogenic and adipogenic differentiation of mesenchymal stem cells. The imbalance between the osteogenic and adipogenic differentiation of MSCs [12] is involved in the occurrence of steroid-related osteonecrosis. PPAR $\gamma$ plays a vital role in modulating differentiation of mesenchymal stem cells, stimulating adipocyte development at the expense of osteoblast differentiation [23]. Hence, PPAR $\gamma$ may serve as a valuable target for methods intended to enhance bone mass. Akune $\mathrm{T}$ et al. demonstrated that heterozygous PPAR $\gamma$-deficient mice exhibit high bone mass and reduced marrow adiposity as well as increased osteoblast number and bone formation rates in mice [24]. In accordance with the findings of Akune $\mathrm{T}$ et al., Gustavo Duque and his colleagues indicated that pharmacological inhibition of PPAR $\gamma$ via BADGE 

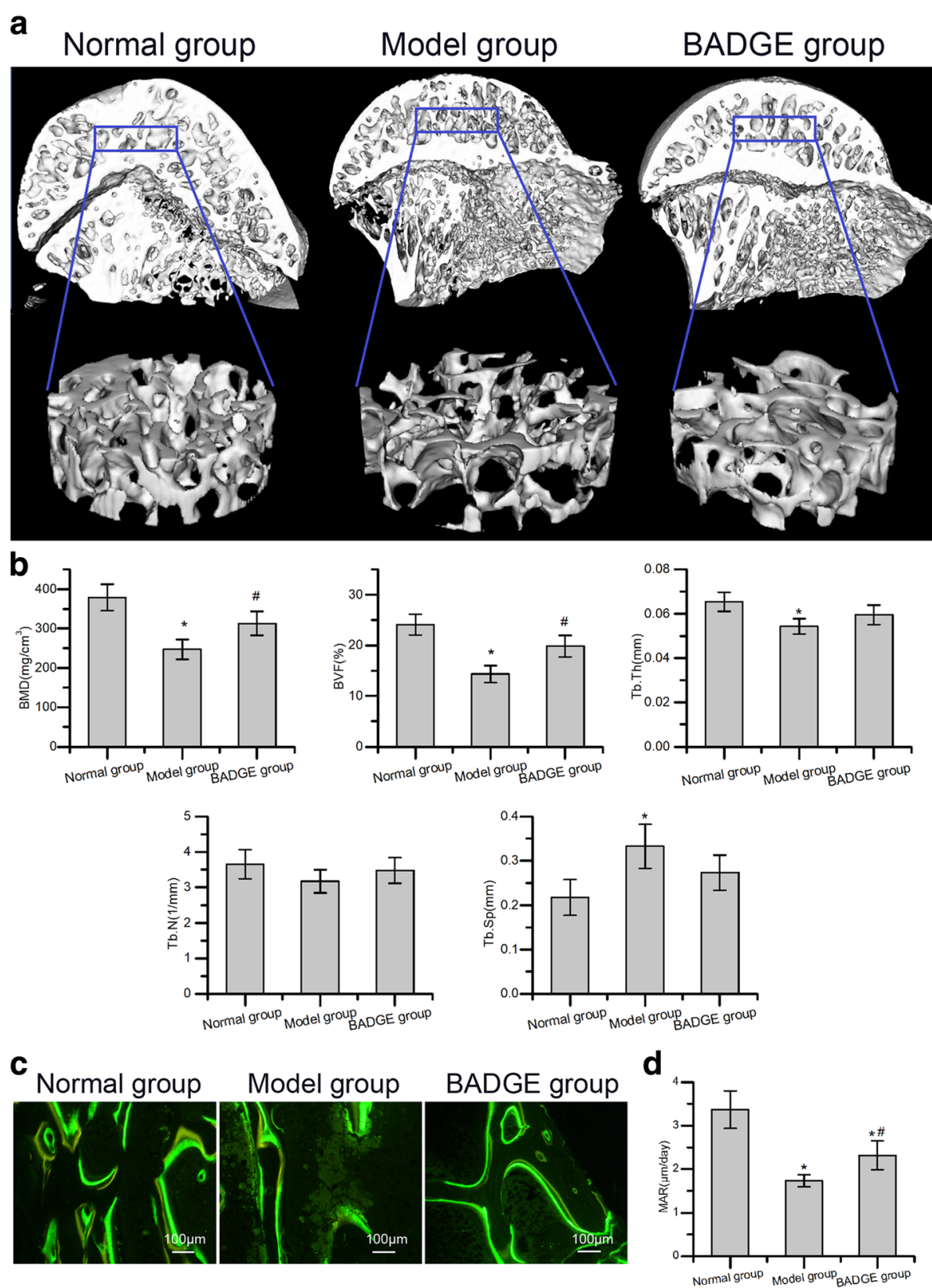

Fig. 3 Treatment with PPARY inhibitors ameliorated microstructural parameters and mineral apposition rate of trabecular bone. a Representative 3-D structure of femoral head of each group 6 weeks after the induction of osteonecrosis. b Quantitative analysis revealed significant reductions in BMD, BVF and Tb.Th and a significant increase in Tb.sp. in the model group compared with the normal group. Compared with the model group, the BADGE group exhibited significantly increased BMD and BVF $(n=5)$. c Representative tetracycline and calcein labelling images. $\mathbf{d}$ Quantitative analysis revealed a significantly increased mineral apposition rate in the BADGE group compared with the model group $(n=5)$. ${ }^{*} P<0.05$ versus normal group; $\# P<0.05$ versus model group

increases bone mass and osteoblastogenesis and stimulates bone formation in male C57BL/6 mice [15]. However, Botolin S and McCabe LR found that pharmacological inhibition of PPAR $\gamma$ by BADGE can not promote expression of osteoblast markers and bone density in diabetic mice [14]. In our study, we explored the effect of BADGE on bone mass, bone formation rates, and expression of osteogenic transcription factors in steroid-related osteonecrosis animal models. Rabbits treated with both BADGE and steroid exhibited increased bone mass and mineral apposition rates. These effects were associated with elevated expression of osteogenic transcription factors, including osteocalcin and Runx2, in the BADGE-treated rabbits. 


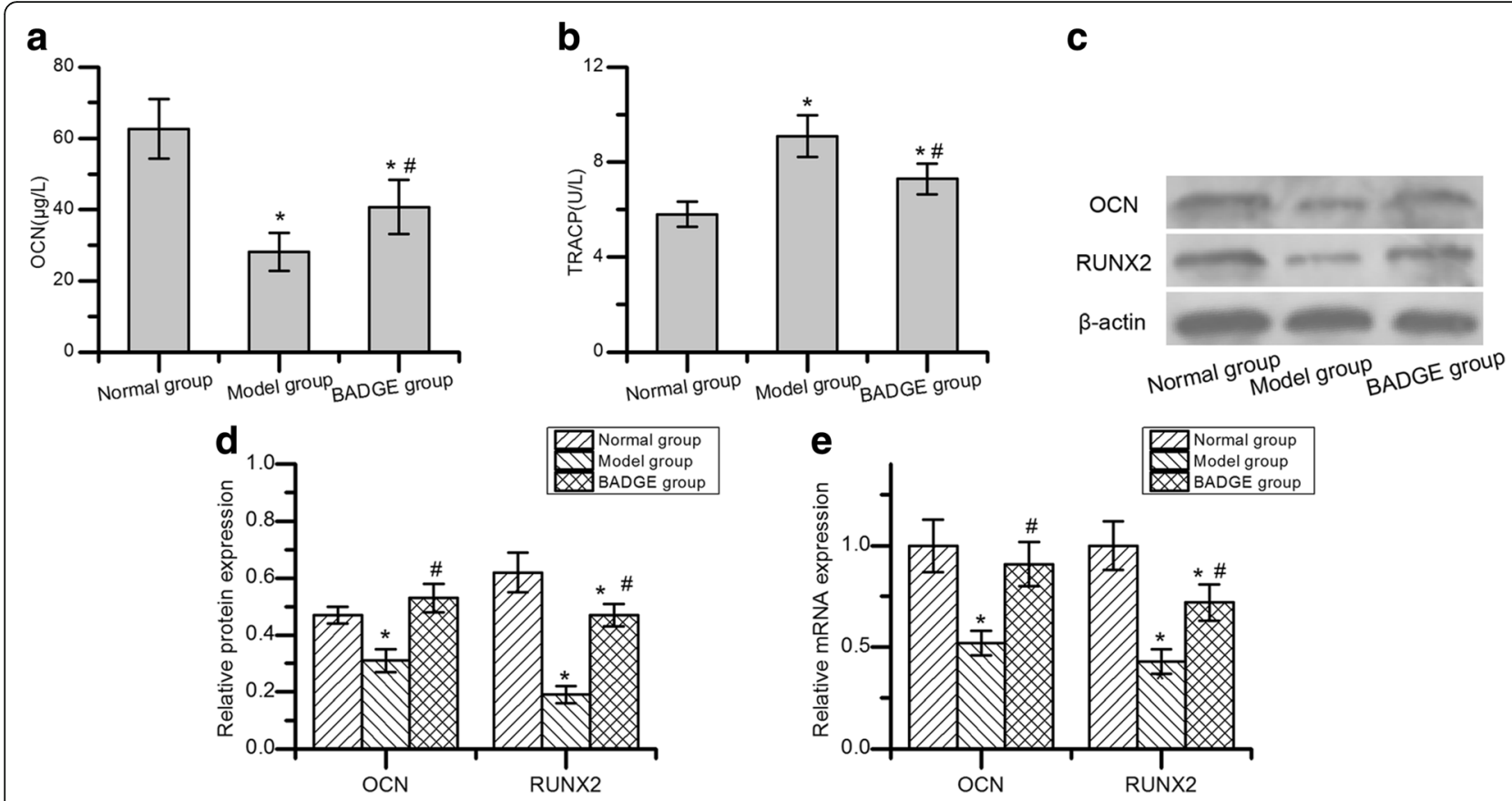

Fig. 4 Treatment with PPARY inhibitors increased the serum osteocalcin (OCN) concentration, decreased the serum TRAP concentration and promoted expression of the osteogenic transcription factors. a The concentration of osteocalcin in the BADGE group was significantly increased compared with the model group. $\mathbf{b}$ In contrast to increased serum osteocalcin, the TRAP concentration was reduced in the BADGE group compared with the model group $(n=5)$. c-e BADGE treatment significantly increased protein and mRNA expression of the osteogenic transcription factors osteocalcin and Runx2 $(n=3) .{ }^{*} P<0.05$ versus normal group; $\# P<0.05$ versus model group

One limitation of the present study was that we limited the therapeutic dose of BADGE to $10 \mathrm{mg} / \mathrm{kg}$. We were unable to determine the optimal level of BADGE. Thus, in the near future, we will conduct additional studies to determine the optimal level of BADGE that can prevent the development of osteonecrosis.

\section{Conclusions}

In summary, our data indicated that PPAR $\gamma$ inhibition by BADGE significantly decreases the incidence of osteonecrosis in steroid-treated rabbits. The present study also provided evidence to suggest that BADGE might prevent steroid-related osteonecrosis by reducing bone marrow adiposity and intraosseous pressure, promoting local blood perfusion, decreasing the expression of adipogenic transcription factors and increasing the expression of osteogenic transcription factors.

\section{Abbreviations}

aP2: Adipocyte fatty acid binding protein 2; BADGE: Bisphenol a diglycidyl ether; BMD: Bone mineral density; BVF: Bone volume fraction; CEBP $\beta$ : CCAAT/ enhancer-binding protein $\beta$; LPS: Lipopolysaccharide; MAR: Mineral appositional rate; MPS: Methylprednisolone; OCN: Steocalcin;

PPARY: Peroxisomal proliferator-activated receptor $\gamma_{i}$ ROI: Region of interest; SI: Signal intensity; Tb.N: Trabecular number; Tb.Sp: Trabecular separation;

Tb.Th: Trabecular thickness; $\mu \mathrm{CT}$ : Micro-computed tomography

\section{Funding}

This work was supported by the Natural Science Foundation of Shaanxi Province, China (Grant No. 2017JQ8022).
Availability of data and materials

The datasets supporting the conclusions of this article are included within the article and available from the corresponding author on reasonable request.

\section{Authors' contributions}

NY and JL made substantial contributions to the conception and design of this manuscript, data acquisition, analysis and interpretation and the drafting of the manuscript. ML and WJ participated in histopathological and biochemical analysis. ZG carried out the western blot analysis and PCR analysis. LF participated in micro-computed tomography analysis and dynamic histomorphometry analysis. KW participated in the measurements of intraosseous pressure. All authors read and approved the final manuscript.

\section{Ethics approval and consent to participate}

This study was carried out upon receiving approval by the Animal Ethical Committee of the Xi'an Jiaotong University was in strict accordance with the NIH Guide for the Care and Use of Laboratory Animals. All efforts were made to minimize suffering.

\section{Competing interests}

The authors declare that they have no competing interests.

\section{Publisher's Note}

Springer Nature remains neutral with regard to jurisdictional claims in published maps and institutional affiliations.

\section{Author details}

${ }^{1}$ Department of Ultrasonography, The First Affiliated Hospital of Xi'an Jiaotong University, Xi'an, Shaanxi 710061, People's Republic of China. ${ }^{2}$ Department of Orthopedics, The First Affiliated Hospital of Xi'an Jiaotong University, 277 West Yanta Road, Xi'an, Shaanxi 710061, People's Republic of China. ${ }^{3}$ Department of Joint Surgery, Honghui Hospital of Xi'an Jiaotong University, Xi'an, Shaanxi 710054, People's Republic of China. ${ }^{4}$ The first 
department of Orthopedics, The Second Affiliated Hospital of Xi'an Jiaotong University, Xi'an, Shaanxi 710004, People's Republic of China.

Received: 11 January 2018 Accepted: 18 April 2018

Published online: 27 April 2018

\section{References}

1. Miyanishi $K$, Yamamoto T, Irisa T, Yamashita A, Jingushi S, Noguchi $Y$, et al. Bone marrow fat cell enlargement and a rise in intraosseous pressure in steroid-treated rabbits with osteonecrosis. Bone. 2002;30:185-90.

2. Jones JP, Jr. Fat embolism, intravascular coagulation, and osteonecrosis. Clin Orthop Relat Res. 1993;(292):294-308.

3. Koo KH, Dussault RG, Kaplan PA, Ahn IO, Kim R, Devine MJ, et al. Fatty marrow conversion of the proximal femoral metaphysis in osteonecrotic hips. Clin Orthop Relat Res. 1999;(361):159-67.

4. Lin L, Dai SD, Fan GY. Glucocorticoid-induced differentiation of primary cultured bone marrow mesenchymal cells into adipocytes is antagonized by exogenous Runx2. Acta Pathol Microbiol Immunol Scand. 2010;118:595-605.

5. Naveiras O, Nardi V, Wenzel PL, Hauschka PV, Fahey F, Daley GQ. Bonemarrow adipocytes as negative regulators of the haematopoietic microenvironment. Nature. 2009;460:259-63.

6. Hozumi A, Osaki M, Goto H, Sakamoto K, Inokuchi S, Shindo H. Bone marrow adipocytes support dexamethasone-induced osteoclast differentiation. Biochem Biophys Res Commun. 2009:382:780-4.

7. Wan Y, Chong LW, Evans RM. PPAR-gamma regulates osteoclastogenesis in mice. Nat Med. 2007:13:1496-503.

8. Lieberman JR. Core decompression for osteonecrosis of the hip. Clin Orthop Relat Res. 2004:(418):29-33.

9. Li J, Li Y, Wang Y, Liu M, Zhao G. Preventive effects of siRNA targeting PPARgamma gene on steroid-induced osteonecrosis in rabbits. Connect Tissue Res. 2014;55:322-30.

10. Jiang Y, Zhang Y, Zhang H, Zhu B, Li P, Lu C, et al. Pravastatin prevents steroidinduced osteonecrosis in rats by suppressing PPARgamma expression and activating Wnt signaling pathway. Exp Biol Med. 2014;239:347-55.

11. Jiang $Y$, Liu D, Kong $X, X u Y$, Chen W, Lin N. Huogu I formula prevents steroid-induced osteonecrosis in rats by down-regulating PPARgamma expression and activating wnt/LRP5/ beta-catenin signaling. J Tradit Chin Med. 2014;34:342-50.

12. Yu Z, Fan L, Li J, Ge Z, Dang X, Wang K. Lithium chloride attenuates the abnormal osteogenic/adipogenic differentiation of bone marrow-derived mesenchymal stem cells obtained from rats with steroid-related osteonecrosis by activating the beta-catenin pathway. Int J Mol Med. 2015; 36:1264-72.

13. Wright HM, Clish CB, Mikami T, Hauser S, Yanagi K, Hiramatsu R, et al. A synthetic antagonist for the peroxisome proliferator-activated receptor gamma inhibits adipocyte differentiation. J Biol Chem. 2000;275:1873-7.

14. Botolin S, McCabe LR. Inhibition of PPARgamma prevents type I diabetic bone marrow adiposity but not bone loss. J Cell Physiol. 2006;209:967-76.

15. Duque G, Li W, Vidal C, Bermeo S, Rivas D, Henderson J. Pharmacological inhibition of PPARgamma increases osteoblastogenesis and bone mass in male C57BL/6 mice. J Bone Miner Res. 2013;28:639-48.

16. Qin L, Zhang G, Sheng H, Yeung KW, Yeung HY, Chan CW, et al. Multiple bioimaging modalities in evaluation of an experimental osteonecrosis induced by a combination of lipopolysaccharide and methylprednisolone. Bone. 2006;39:863-71.

17. Yamamoto T, Irisa T, Sugioka Y, Sueishi K. Effects of pulse methylprednisolone on bone and marrow tissues: corticosteroid-induced osteonecrosis in rabbits. Arthritis Rheum. 1997;40(11):2055-64

18. Kuribayashi M, Fujioka M, Takahashi KA, Arai Y, Ishida M, Goto T, et al. Vitamin E prevents steroid-induced osteonecrosis in rabbits. Acta Orthop. 2010;81:154-60

19. Pengde $K$, Fuxing $P$, Bin $S$, Jing $Y$, Jingqiu C. Lovastatin inhibits adipogenesis and prevents osteonecrosis in steroid-treated rabbits. Joint Bone Spine. 2008;75:696-701.

20. Li J, Fan L, Yu Z, Dang X, Wang K. The effect of deferoxamine on angiogenesis and bone repair in steroid-induced osteonecrosis of rabbit femoral heads. Exp Biol Med. 2015;240:273-80

21. Wu X, Yang S, Duan D, Zhang Y, Wang J. Experimental osteonecrosis induced by a combination of low-dose lipopolysaccharide and high-dose methylprednisolone in rabbits. Joint Bone Spine. 2008;75:573-8.
22. Zhang $G$, Qin $L$, Sheng $H$, Wang $X L$, Wang $Y X$, Yeung DK, et al. A novel semisynthesized small molecule icaritin reduces incidence of steroidassociated osteonecrosis with inhibition of both thrombosis and lipiddeposition in a dose-dependent manner. Bone. 2009:44:345-56.

23. Kim J, Ko JA. Novel PPARgamma2 modulator sLZIP controls the balance between adipogenesis and osteogenesis during mesenchymal stem cell differentiation. Cell Death Differ. 2014;21:1642-55.

24. Akune T, Ohba S, Kamekura S, Yamaguchi M, Chung UI, Kubota N, et al. PPARgamma insufficiency enhances osteogenesis through osteoblast formation from bone marrow progenitors. J Clin Invest. 2004;113:846-55.

\section{Ready to submit your research? Choose BMC and benefit from:}

- fast, convenient online submission

- thorough peer review by experienced researchers in your field

- rapid publication on acceptance

- support for research data, including large and complex data types

- gold Open Access which fosters wider collaboration and increased citations

- maximum visibility for your research: over $100 \mathrm{M}$ website views per year

At BMC, research is always in progress.

Learn more biomedcentral.com/submissions 